\title{
Antidyskinetic Treatment with the mGluR5 Antagonist MPEP Affects CaMKII/CREB/BDNF Molecular Pathways in the Parkinsonian Striatum
}

\section{Yixian Huang}

Second Affiliated Hospital of Soochow University

\section{Qilin Zhang}

Second Affiliated Hospital of Soochow University

zhoubing zhan

Second Affiliated Hospital of Soochow University

Haiyang Shu

Second Affiliated Hospital of Soochow University

Junyan Zhao

Second Affiliated Hospital of Soochow University

Tili Zhen

Second Affiliated Hospital of Soochow University

Weifeng Luo ( $\square$ lwfwxx@126.com )

Second Affiliated Hospital of Soochow University https://orcid.org/0000-0002-3594-1885

\section{Research}

Keywords: Parkinson's disease, L-DOPA-induced dyskinesia, mGluR5, MPEP, Molecular Pathways

Posted Date: April 17th, 2020

DOl: https://doi.org/10.21203/rs.3.rs-20433/v1

License: (c) (i) This work is licensed under a Creative Commons Attribution 4.0 International License. Read Full License 


\section{Abstract \\ Background}

L-DOPA is still the gold-standard drug for the treatment of Parkinson's disease (PD). However, the longterm therapy often causes L-DOPA-induced dyskinesia (LID). Metabotropic glutamate receptor type 5 (mGluR5) is abundant in the basal ganglia, and its antagonists is thought to alleviates LID, but the underlying mechanisms have remained unclear.

\section{Methods}

We used 6-hydroxydopamine-lesioned rats to create PD rat model, PD rats were daily treated with L-DOPA alone or with MPEP 30 min before L-DOPA for 3 weeks, and at least 21 days of L-DOPA was administrated followed with microinjection of saline, CaMKII antagonist KN-93, anti-CREB, or anti-BDNF into the lesioned striatum of all the PD rats. The behavioral evaluation of abnormal involuntary movements(AIM) and rotational behavior tests were performed on the 2, 9, 11, 18, 21 and 23 days after drug application, and to tested the protein level of mGluR5, CaMKII, CREB and BDNF by western blot.

\section{Results}

Our results showed that MPEP cotreatment attenuates the abnormal involuntary movements, reversed the reduction of rotational response duration, and reduced overexpression of striatal mGluR5 and CaMKII/CREB/BDNF in the LID rats. Furthermore, we found that the CaMKII inhibitor KN-93, anti-CREB and anti-BDNF intrastriatal injection partly attenuates LID; KN-93 downregulated the striatal p-CaMKII, pCREB and BDNF expression of the lesioned side, but the striatal mGluR5 expression without inhibition; anti-CREB downregulated the striatal p-CREB and BDNF expression of the lesioned side, but the striatal mGluR5 and p-CaMKII expression without inhibition; anti-BDNF downregulated the striatal BDNF expression of the lesioned side, but the mGluR5, CaMKII and CREB protein without inhibition.

\section{Conclusions}

These fundings suggested that mGluR5 specific antagonist MPEP attenuates L-DOPA-induced dyskinesia through affects CaMKII/CREB/BDNF molecular pathways in the striatum in this LID rat model.

\section{Introduction}

Parkinson's disease (PD), one of most common neurodegenerative diseases, the loss of dopaminergic neurons in the substantia nigra pars compacta are main neuropathological features, resulting in significantly decreased dopamine levels in the striatum (Bastide et al., 2015). Although L-DOPA is still the gold-standard drug of dopamine replacement treatment for PD, however, the long-term L-DOPA therapy 
causes a decrease in the efficacy and disabling abnormal involuntary movement (AIM), namely L-DOPAinduced dyskinesias (LID), which greatly hampered the use of L-DOPA in PD treatment (Fabbrini et al., 2007). At present, the underlying molecular mechanisms of LID are still elusive. Several neurotransmitter systems in the local striatum have been implicated in the pathogenesis of LID. It is known that overactivation of glutamatergic signaling and the hypersensitivity of the glutamatergic system in the basal ganglia play an important role in the pathogenesis of LID (Litim et al., 2017). Several researchers reported that the blockade of the metabotropic glutamate receptor 5 (mGluR5) could attenuate the LID (Dekundy et al., 2006; Mela et al., 2007; Gravius et al., 2008; Levandis et al., 2008), emerging evidence has come to support the important role of mGluR5 in the development of LID (Johnston et al., 2010; Morin et al., 2010; Rylander et al., 2010; Gregoire et al., 2011; Rascol et al., 2014), and the pharmacological inhibition of the mGluR5 with a specific antagonist MPEP (2-Methyl-6-(phenylethynyl)-pyridine) reduced the L-DOPA-induced expression of FosB, Preprodynorphin, GAD, PSD95, SAP (Levandis et al., 2008; Morin et al., 2010; Morin et al., 2013a; Huang et al., 2018). However, the underlying molecular mechanisms of the antidyskinetic activity of mGluR5 antagonists remain largely unknown.

$\mathrm{Ca}^{2+} /$ calmodulin-dependent protein kinase II (CaMKII) is ubiquitously expressed in the central nervous system and is particularly abundant at excitatory synapses. This kinase plays an important role in the regulation of neuronal survival and death (Zhang et al., 2017b). CaMKIl and its interacting partners are also believed to play a critical role in the pathogenesis of various neurological and neurodegenerative disorders, such as PD. Recent research show that chronic L-DOPA treatment activates CaMKII in striatal neurons (Gan et al, 2015), and the pharmacological inhibition of the CaMKII with a selective inhibitor KN93 could ameliorated dyskinesia in a rat PD model (Zhang et al., 2014; Yang et al, 2018). However, the specific molecular mechanisms of the involvement of CaMKII in LID are not fully unclear. CAMP-response element binding protein (CREB) is another intracellular signaling that its activation prevents dopaminergic cell death in PD (Ham et al., 2017). Innovative treatment strategies for PD, especially at its early stages, have gained paramount clinical attention (Beitz, et al., 2014). Brain-derived neurotrophic factor (BDNF), a member of the nerve growth factor family of proteins, is a major promoter of synaptic plasticity, and regulates neuronal proliferation, differentiation and survival (Binder and Scharfman, 2004; Cohen-Cory et al., 2010; Park and Poo, 2013). The recent research indicates that L-DOPA administration induces BDNF release from corticostriatal fibers, which in-turn increases the expression of D3 receptors, and the D3 expression increase plays a key role in the emergence of LID (Guillin et al., 2001). Furthermore, recent findings demonstrate that repeated L-DOPA therapy enhanced the levels of $c$-fos and BDNF mRNA in the dopamine-depleted subthalamic nucleus in the PD model rat (Zhang et al., 2006).

It is of great importance to investigate what the molecular alterations in the mGluR5-related signaling pathway are, in order to interpret the antidyskinesia effect of the antagonists of mGluR5. This study aimed to investigate the effects of mGluR5 specific antagonist MPEP on LID, and to tested the protein level of mGluR5, CaMKII, CREB and BDNF in the mGluR5 mediated CaMKII/CREB/BDNF signaling pathway. 


\section{Materials And Methods}

\section{Animals}

Male adult Sprague-Dawley animals (weighing 180-220 g) were obtained from the Center for Experimental Animals, Soochow University,China. The maintenance of the animals followed the guidelines of the National Institutes of Health for the care and use of laboratory animals. All experimental protocols involving animals were approved by the ethics committee of the Second Affiliated Hospital of Soochow University.

\section{Reagents}

6-OHDA(6-hydroxydopamine), ascorbic acid, L-DOPA methyl ester, benserazide, MPEP (2-methyl-6(phenylethynyl)pyridine), CaMKII antagonist KN-93, anti-CREB and anti-BDNF were purchased from Sigma-Aldrich (St Louis, MO). Apomorphine was purchased from Tocris (Bristol, UK). Rabbit monoclonal antibody against the mGluR5, CaMKII, CREB and BDNF receptor was obtained from CST (Cell Signaling Technology, USA), anti- $\beta$-actin antibody was purchased from Beyotime Biotechnology (Shanghai, China).

\section{6-OHDA lesion and screening of rotational responses}

To establish 6-OHDA-induced parkinsonian model, the animals were anesthetized with $10 \%$ chloral hydrate $(0.5 \mathrm{ml} / 100 \mathrm{~g})$ deeply and immobilized in a stereotaxic apparatus (David Kopf Instruments, Tujunga, CA). Based on an adult rat brain atlas (Paxinos and Watson, 1986), $8 \mu \mathrm{g}$ of 6-OHDA (dissolved in a volume of $4 \mu \mathrm{l}$ saline containing $0.02 \%$ ascorbic acid) was injected unilaterally into the right medial forebrain bundle (leading to nigrostriatal lesion) with a 10- $\mu$ l Hamilton syringe at the two following coordinates: (1) AP,-1.8 mm; L, -2.5 mm; DV,-8.0 mm from Bregma. (2) AP, - $1.8 \mathrm{~mm} ; \mathrm{L},-2.5 \mathrm{~mm}$; DV, $-7.5 \mathrm{~mm}$ from Bregma. A total of $16 \mu \mathrm{g} 6-\mathrm{OHDA}$ were injected at the two coordinates ( $8 \mu \mathrm{l}$ for each site). 6-OHDA was freshly prepared in the dark to prevent autoxidation, and delivered at a rate of $0.5 \mu \mathrm{l} / \mathrm{min}$ by a 10- $\mu$ micro-injector. Before slowly withdrawal, the syringe was retained for about 5 min to allow for diffusion of the toxin and to avoid toxin reflux. After two weeks, the lesioned animals were delivered with apomorphine at a dose of $0.25 \mathrm{mg} / \mathrm{kg}$ (dissolved in physiological saline, i.p.) and were kept in a $40 \mathrm{~cm}$ diameter round kettle. The numbers of contralateral rotations of each rat were recorded visually within $30 \mathrm{~min}$; the animals with over 7 contralateral turns within one minute were considered as Parkinson model rats and chosen for further experiments.

\section{The Design Of Experiments}

Two weeks following apomorphine detection, successfully induced PD model animals were randomly classified into four groups ( $n=10$ for each group): (1) Intraperitoneal (i.p.) injection with saline (the control group); (2) administration of L-DOPA (25 mg/kg L-DOPA plus $6.25 \mathrm{mg} / \mathrm{kg}$ benserazide in saline, 
the L-DOPA group); (3) i.p. injection of MPEP ( $1.5 \mathrm{mg} / \mathrm{kg}$ in saline) $30 \mathrm{~min}$ before an injection of $25 \mathrm{mg} / \mathrm{kg}$ L-DOPA plus $6.25 \mathrm{mg} / \mathrm{kg}$ benserazide ( the co-administration group) ; (4) i.p. injection of MPEP (1.5 mg/kg in saline, the MPEP group). Above four groups were all administrated for 21 days. The dose of L-DOPA was selected according to our previous study (Huang et al., 2011), while the dose for MPEP was used based on another study (Levandis et al., 2008).

Moreover, Fifty successfully PD model rats were randomly classified into five groups ( $n=10$ for each group): (1) Intraperitoneal (i.p.) injection with saline (the control group) for 23 days $(n=10)$. The other forty rats were treated with L-DOPA ( $25 \mathrm{mg} / \mathrm{kg} \mathrm{L-DOPA} \mathrm{plus} 6.25 \mathrm{mg} / \mathrm{kg}$ benserazide in saline) to 21 days, at the 22 day, L-DOPA-treated and dyskinetic rats were randomly divided into four groups $(\mathrm{n}=10$ for each group), these rats received intrastriatal administration of (2) saline, (3) CaMKII antagonist KN-93, (4) antiCREB, or (5) anti-BDNF. For intrastriatal injection, rats were anesthestized by $10 \%$ chloral hydrate

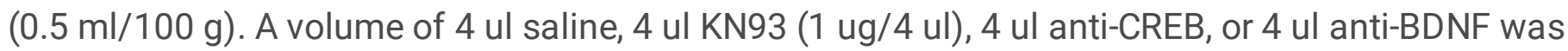
injected at the coordinates: $\mathrm{AP}=0.5 \mathrm{~mm}$ from bregma; $\mathrm{ML}=-2.5 \mathrm{~mm}$ from midline; and $\mathrm{DV}=-4.6$ from the dura surface. A microsyringe was kept in place for an additional $5 \mathrm{~min}$ before being retracted slowly. Rats were left on a warm plate after surgery to avoid hypothermia until recovery, at 23 day, rats were treated with L-DOPA.

\section{Behavioral Tests}

The record of abnormal involuntary movements (AIM) triggered by L-DOPA was visually collected during behavioral tests (Lundblad et al., 2002) and divided into three kinds: (1) axial AIM: dystonic posture of the upper part of the body toward the un-lesioned side; (2) limb AIM: movements from the forelimb and the paw towards the un-lesion side; (3) orolingual AIM: jaw movements and tongue protrusion. Each kind was scaled based on a severity ranged 0 to 4 : 0 indicates lack of AIM; 1 indicates existence during less than half one minute; 2 indicates existence during over half one minute; 3 indicates persistent existence but inhibited by external stimuli; 4 indicates persistent existence without the inhibitory possibility. during 140 minutes after L-DOPA administration, rats were assessed every 20 minutes individually. Each rat was rated for appearance of abnormal movements in axial, limb, orolingual movements. Each AIM was quantified within one minute and present as time which the rat stayed for each movement. The total scores of axial, limb and orolingual AIM were regarded as ALO AIM scores.

Rotational behavior was measured immediately following the treatment of L-DOPA. The duration of the rotational response was considered the time between the first 5 min interval when turning exceeded $20 \%$ of the peak rate and the first interval when turning fell below $20 \%$ of the peak rate. The peak intensity of rotation was measured as the peak number of contralateral turns in any 5 min interval.

The behavioral evaluation of AIM, and rotational behavior tests were performed on the 2, 9, 11, 18 and 21 days after L-DOPA application. The AIM evaluation was performed on the 21 and 23 days after drug administration. 


\section{Western Blotting}

Rats were anesthetized with $10 \%$ chloral hydrate $(0.5 \mathrm{ml} / 100 \mathrm{~g})$ for 30 min after the last administration, rats were sacrificed and their brains were quickly taken out. Then the lesioned striatum were separated and stored at $-70^{\circ} \mathrm{C}$ for Western blots. The tissues from the dorsal striatum were homogenized and centrifuged at $12000 \mathrm{~g}$ for $30 \mathrm{~min}$. The protein concentration was determined by a BCA assay kit (Pierce Chemical, Rockford, IL). A total of $60 \mu \mathrm{g}$ of protein lysates from each sample were added into the loading buffer, mixed and heated at $95^{\circ} \mathrm{C}$ for $5 \mathrm{~min}$. Then the samples were loaded into SDS polyacrylamide electrophoresis gels (8\%) and transferred to PVDF membranes (Bio-Rad, Hercules, CA). The resultant blots were blocked in Tris buffered saline/Tween 20 (TBST) buffer $(10 \mathrm{mmol} / \mathrm{L}$ Tris, $150 \mathrm{mmol} / \mathrm{L} \mathrm{NaCl}$,

$0.1 \%$ Tween-20, $\mathrm{pH} 8.0$ ) with $5 \%$ milk for $1 \mathrm{~h}$. The membranes were then incubated with the indicated primary antibodies (rabbit anti-mGluR5, 1:2000, or rabbit anti-P-CaMKII/CaMKII, 1:1000, or rabbit anti-PCREB/CREB, 1:1000, or rabbit anti-BDNF, 1:2000, or mouse anti- $\beta$-actin, 1:5000) at $4{ }^{\circ} \mathrm{C}$ overnight. Next, the membranes were rinsed with TBS-T for three times and incubated with appropriate secondary antibodies for $1 \mathrm{~h}$ at room temperature. At last, the membrane was briefly washed and subjected to ECL chemiluminescence exposure (GE healthcare, Buckinghamshire, UK). Images were obtained on a computer-aided CCD camera. Optical density (OD) was detected with Mercator software (Explora Nova). All individual protein bands were compared with their internal control actin values in order to provide relative protein abundance. All the procedures were repeated 3 times.

\section{Statistical analysis}

All data were expressed as mean \pm standard error of the mean (SEM). Behavioral assessments and biochemical data analysis among different groups were performed using one-way analysis of variance (ANOVA) coupled with the LSD's post hoc analysis (SPSS, 18.0, Chicago, IL). $P<0.05$ was viewed as statistical significance.

\section{Results}

\section{MPEP attenuates abnormal involuntary movements induced by L-DOPA}

To mimic dyskinesia phenomena in PD patients, we consecutively treated PD model rats with L-DOPA for 21 days, and observed progressive AIM. Whereafter, we examined the potential role of mGluR5 in AIM. By co-treatment of L-DOPA with the specific mGluR5 antagonist MPEP, we found the alleviation of these behavioral abnormalities. Specifically, at 21 day after L-DOPA administration, the axial AIM score of MPEP plus L-DOPA group was significantly decreased than that of L-DOPA treatment group $(P<0.05$, Fig. 1a). Similarly, the limb AIM score of the coadministration group was markedly reduced at 18 and 21 day as compared to the L-DOPA treatment group $(P<0.01$, Fig. $1 \mathrm{~b})$. Moreover, the orolingual AIM score of the coadministration group was significantly decreased at 21 day as compared to the L-DOPA 
treatment group ( $P<0.01$, Fig. 1c). Interestingly, the total AIM score of the coadministration group were reduced at 18 and 21 day as compared to the L-DOPA treatment group $(P<0.05$, Fig. $1 d)$. Lesioned rats with received of saline (controls, $n=10)$ or MPEP $(n=10)$ alone failed to develop AIM. Take together, our results indicated that inhibition of mGluR5 by MPEP prevents L-DOPA-induced AIM in 6-OHDA-lesioned rats.

\section{MPEP ameliorated the reduction of rotational response duration induced by L-DOPA}

To mimic the "wearing-off" phenomena in PD patients, we consecutively treated PD model rats with LDOPA for 21 days, and observed progressive shortening in the duration of rotational response. Our results revealed that administration of L-DOPA alone, given twice daily for 21 days, significantly reduced the rats' rotational response duration; the duration of the rotational response reduced from $138.5 \pm 3.6 \mathrm{~min}$ to $101.5 \pm 5.1$ min during the 21-day treatment period $(F=7.991, F=13.833, F=35.661, P<0.05)$ (Fig. 2$)$. By contrast, coapplication of MPEP with L-DOPA maintained the rotational response duration throughout the 21-day treatment period $(P>0.05)$ (Fig. 2). This effect was maintained until the end of MPEP with L-DOPA administration and the rotational duration of four time points were not different during the course of the treatment, as compared to L-DOPA treatment group at day $2(P>0.05)$ (Fig. 2). Lesioned rats that injection saline (controls, $n=10)$ or MPEP $(n=10)$ alone did not develop rotational response at all.

\section{MPEP attenuates the upregulation of striatal mGluR5, CaMKII, CREB and BDNF expression induced by L- DOPA}

Our above behavioral results suggested the overactivity of mGluR5 expression in PD model rats treated with L-DOPA. In addition, there is evidence that overexpression of mGluR5 in the basal ganglia of PD model monkeys with LID and in PD patients with motor complications (Samadi et al., 2008; Ouattara et al., 2010; Ouattara et al., 2011; Morin et al., 2013a, c). Whereafter, we examined the expression of mGluR5, CaMKII, CREB and BDNF at the striatum. Western blot analysis revealed that delivery with L-DOPA upregulated the striatal mGluR5, $p$-CaMKII, $p$-CREB and BDNF expression in the lesioned side $(P<0.05$, Fig. 3). The up-regulation was largely inhibited by coadministration with MPEP $(P<0.05$, Fig. 3). Collectively, our results suggested overexpression of mGluR5, $p$-CaMKII, $p$-CREB and BDNF in PD model rats and the inhibitory role of MPEP.

\section{$\mathrm{KN}-93$, anti-CREB, anti-BDNF attenuates L-DOPA-induced dyskinesia and affects striatal CaMKIIICREB/BDNF expression}

Our behavioral results and western blot analysis suggested LID may be due to mGluR5-related higher CaMKII/CREB/BDNF expression. However, how these abnormal molecular pathways alterations influence the contribution of mGluR5 to LID needs to be further investigated. We consecutively treated PD model rats with L-DOPA for 21 days, and observed progressive AIM. By treatment of the specific CaMKII inhibitor $\mathrm{KN}-93$, anti-CREB, anti-BDNF at the 22 day, we found the alleviation of these behavioral abnormalities. Specifically, at 23 day after L-DOPA application, the axial AIM score of anti-CREB and anti-BDNF groups were reduced than that of 21 day $(P<0.05$, Fig. 4a). Similarly, the limb AIM score of KN-93 and anti-BDNF 
groups were significantly reduced than that of 21 day $(P<0.05$, Fig. $4 b)$. Moreover, the orolingual AIM score of the $\mathrm{KN}-93$ and anti-CREB groups were decreased at 23 day as compared to 21 day $(P<0.05$, Fig. 4c). Interestingly, the total AIM score of the KN-93 and anti-CREB groups were significantly decreased on the 23 day, as compared to 21 day $(P<0.05$, Fig. 4 d). Nevertheless, the limb, orolingual and total AIM score of the L-DOPA group was significantly increased at 23 day as compared to 21 day $(P<0.05$, Fig. 4). In addition, we further examined that the striatal mGluR5 expression of the L-DOPA, KN-93, anti-CREB and anti-BDNF groups were upregulated as compared to control group $(P<0.05$, Fig. 5a,b). CaMKII inhibitor $\mathrm{KN}$-93 downregulated the striatal $\mathrm{p}$-CaMKII, $\mathrm{p}$-CREB and BDNF protein expression of the lesioned side, but the mGluR5 expression without inhibition $(P<0.05$, Fig. 5a,c). CREB inhibitor anti-CREB downregulated the striatal p-CREB and BDNF protein expression of the lesioned side, but the striatal mGluR5 and pCaMKII expression without inhibition $(P<0.05$, Fig. 5a,d). BDNF inhibitor anti-BDNF downregulated the striatal BDNF expression of the lesioned side, but the mGluR5, CaMKII and CREB expression without inhibition $(P<0.05$, Fig. 5a,e). Collectively, these data suggested that the mGluR5 antagonist MPEP attenuates L-DOPA-induced dyskinesia through affects CaMKII/CREB/BDNF molecular pathways in the Parkinsonian striatum.

\section{Discussion}

Glutamate plays a key role in the mammalian brain, serving as most of synaptic transmission in the central nervous system (Meldrum et al., 2000; Platt et al., 2007). It was demonstrated that hypersensitivity of the glutamatergic system and the overactivation of glutamatergic transmission in the basal ganglia play an important role in the pathophysiology of PD (Klockgether and Turski, 1993) and LID (Chase and Oh, 2000; Calon et al., 2003b). There is reported that the therapeutic use of an mGluR5 antagonist led to normalization of glutamate neurotransmission in the PD brain and prevented the occurrence of LID (Morin et al., 2013c). Consistent with the notion, our results confirmed that antagonizing mGluR5 reduced the AIM score in the PD rat model and MPEP strengthened L-DOPA anti-parkinsonian effects. In this present study, we explored the possible molecular mechanism of the alleviation effect mediated by the antagonist of mGluR5. Our results showed that motor complications induced by administration of LDOPA are companied by the signaling proteins in 6-OHDA-lesioned hemiparkinsonian rats, while MPEP cotreatment reduced overexpression of striatal mGluR5 and CaMKII/CREB/BDNF in the LID rats. In addition, our results further demonstrated that the striatal mGluR5 expression of the L-DOPA, KN-93, antiCREB and anti-BDNF groups were remarkably upregulated as compared to control group. KN-93, antiCREB and anti-BDNF intrastriatal injection partly attenuates LID. CaMKII inhibitor KN-93 downregulated the striatal p-CaMKII, $\mathrm{p}$-CREB and BDNF expression of the lesioned side, but the mGluR5 expression without inhibition. anti-CREB downregulated the striatal p-CREB and BDNF protein expression of the lesioned side, but the striatal mGluR5 and p-CaMKII protein expression without inhibition. anti-BDNF downregulated the striatal BDNF expression of the lesioned side, but the mGluR5, CaMKII and CREB expression without inhibition. Taken together, our results provide evidence that mGluR5 specific antagonist MPEP attenuates L-DOPA-induced dyskinesia through affects CaMKII/CREB/BDNF molecular pathways in this experimental model of LID. 
The 6-OHDA or MPTP-lesioned PD model animals administrated with L-DOPA induced AIM, equivalent to the "peak-dose" dyskinesia in L-DOPA-treated PD patients (Cenci et al., 2006). Our results showed that the rats treated with L-DOPA alone displayed onset and progressive increase of AIM, whereas, cotreatment of MPEP with L-DOPA partly decreased axial, orolingual, limb and total AIM scores. Consistently, several reports indicating that selective mGluR5 antagonist MPEP blocked the occurrence of LID and kept antiparkinsonian effects in 6-OHDA-lesioned rats or MPTP-treated monkeys (Morin et al., 2010; Morin et al., 2013a). Moreover, our results showed that the CaMKII inhibitor KN-93, CREB inhibitor and BDNF inhibitor partly decreased axial, orolingual, limb, and total AIM scores in the 6-OHDA-lesioned rats treated with L-DOPA exhibited AIM.

L-DOPA treatment induced a progressive shortening in the duration of rotational response in 6-OHDAlesioned PD model rats, mimicking the "wearing-off" phenomena in PD patients (Henry et al., 1998; Oh and Chase, 2002). Our results showed that the rats administrated with L-DOPA induced a progressive shortening in the duration of rotational response, while cotreatment of MPEP with L-DOPA for 21 day ameliorated the duration of rotational response. Furthermore, the results have provided indications as to the satisfactory tolerability in the effect of MPEP, as the amelioration was sustained even after the MPEP application. Our results are in accord with a recent study showing that AFQ056 tested with L-DOPA maintained the antiparkinsonian effect and increased locomotion. in MPTP-lesioned PD monkeys (Grégoire et al., 2011).

The underlying molecular mechanisms of the anti-dyskinetic activity of mGluR5 antagonists remain largely unknown. Our results showed that cotreatment of MPEP with L-DOPA to PD rats not only alleviated AIM (equivalent to motor complications induced by L-DOPA in human), but also decreased overexpression of mGluR5 in the striatum, consistent with a previous report (Morin et al., 2013b) that mGlu5 receptor specific bindings in the basal ganglia were boosted in L-DOPA-administrated MPTP monkeys as compared to controls but not in the animals cotreated with MPEP and L-DOPA. Interestingly, MPEP cotreatment with L-DOPA reduced overexpression of striatal CaMKII/CREB/BDNF in the LID rats, comparable to the administration of L-DOPA alone group.

Our study showed that LID may be due to mGluR5-related higher CaMKII/CREB/BDNF expression. However, how these abnormal molecular pathways alterations influence the contribution of mGluR5 to LID needs to be further investigated. By using behavioral tests and western blot analysis, we examined the effects of specific CaMKII inhibitor KN-93, anti-CREB, and anti-BDNF on LID and the markers of the mGluR5-related CaMKII/CREB/BDNF signaling pathway in the PD model rats. Our data showed that KN93, anti-CREB and anti-BDNF intrastriatal injection partly attenuates LID, and accordingly downregulated the striatal p-CaMKII, p-CREB and BDNF protein expression of the lesioned side, while mGluR5 protein expression without inhibition. Taken together, these findings suggested that the mGluR5 specific antagonist MPEP attenuates L-DOPA-induced dyskinesia through affects CaMKII/CREB/BDNF molecular pathways in this experimental model of LID. However, further studies, using striatal-specific CaMKIIICREB/BDNF KO or viral-delivered RNAi against CaMKII/CREB/BDNF in this region, are obviously 
needed to resolve whether the increase in CaMKII/CREB/BDNF expression reflects therapeutic actions of L-DOPA or the propensity of L-DOPA to cause side effects.

\section{Conclusions}

In summary, this study suggested that the selective mGluR5 antagonist MPEP alleviated the abnormal involuntary movements, reversed the reduction of rotational response duration, and inhibited the overexpression of striatal mGluR5 and CaMKII/CREB/BDNF induced by L-DOPA treatment rats in the lesioned striatum. Furthermore, these results provide evidence that $\mathrm{KN}-93$, anti-CREB and anti-BDNF partly attenuates AIM, accordingly inhibited the hyperactivity of striatal CaMKII/CREB/BDNF protein expression in the lesioned striatum of LID rats. Therefore, these results suggested that mGluR5 specific antagonist MPEP attenuates L-DOPA-induced dyskinesia through affects CaMKII-CREB-BDNF molecular pathways in the striatum in this LID rat model.

\section{Abbreviations}

PD

Parkinson's disease

6-OHDA

6-hydroxydopamine

LID

L-DOPA-induced dyskinesia

mGluR5

metabotropic glutamate receptor 5

AIM

Abnormal involuntary movement

MPEP

2-Methyl-6-(phenylethynyl)-pyridine

CaMKII

$\mathrm{Ca} 2+/$ calmodulin-dependent protein kinase II

\section{Declarations}

\section{Ethics approval and consent to participate}

All experimental protocols involving animals were approved by the ethics committee of the Second Affiliated Hospital of Soochow University.

\section{Consent for publication}

Not applicable. 
Availability of data and materials

The data that support the findings of this study are available from the corresponding author, upon reasonable request.

\section{Competing interests}

The authors declare that they have no competing interests.

\section{Funding}

This work was funded by National Natural Science Foundation of China (N0.81200970), Suzhou Sci \& Tech Program Grant (SYSD2018098) and Changzhou High-Level Medical Talents Training Project (NO.2016CZBJ023).

\section{Author's contributions}

Yixian Huang, Qilin Zhang, Zhoubing zhan and Weifeng Luo were involved in the study design, Animal experiment, Molecular biology experiment, data collection, data interpretation data management and data analysis and contributed equally to the first draft. Junyan Zhao, Tili Zhen, and Haiyang Shu participated in the Animal experiment, Molecular biology experiment, data collection. They provided critical review of the manuscript. All authors read and approved the final manuscript.

\section{Acknowledgments}

Not applicable

\section{Author information}

\section{Affiliations}

Department of Neurology, The Second Affiliated Hospital of Soochow University, Suzhou, China.

Yixian Huang, Qilin Zhang, Junyan Zhao, Tili Zhen, Weifeng Luo

Institute of Neuroscience, Soochow University, Suzhou China.

Weifeng Luo

Department of Nephrology, The Second Affiliated Hospital of Soochow University, Suzhou, China.

Zhoubing zhan

Department of Neurology, Taikang People's Hospital of Henan Province, No. 469 Southern Jianshe Road, 461400, Taikang, China. 


\section{References}

1. Bastide MF, Meissner WG, Picconi B, Fasano S, Fernagut PO, Feyder M, Francardo V, Alcacer C, Ding Y, Brambilla R, Fisone G, Jon Stoessl A, Bourdenx M, Engeln M, Navailles S, De Deurwaerdere P, Ko WK, Simola N, Morelli M, Groc L, Rodriguez MC, Gurevich EV, Quik M, Morari M, Mellone M, Gardoni F, Tronci E, Guehl D, Tison F, Crossman AR, Kang UJ, Steece-Collier K, Fox S, Carta M, Cenci A. M., Bezard, E. Pathophysiology of L-dopa-induced motor and non-motor complications in Parkinson's disease. Prog Neurobiol. 2015;132:96-168.

2. Beitz JM. Parkinson's disease: a review. Front Biosci. 2014;6(1):65-74.

3. Binder DK. Brain-derived neurotrophic factor. Growth Factors. 2004;22(3):123.

4. Calon F, Rajput AH, Hornykiewicz O, Bedard PJ, Di Paolo T. Levodopa induced motor complications are associated with alterations of glutamate receptors in Parkinson's disease. Neurobiol Dis. 2003b;14:404-16.

5. Cenci MA, Lundblad M. Post-versus presynaptic plasticity in L-DOPA-induced dyskinesia. J Neurochem. 2006;99:381-92.

6. Chase TN, Oh JD. Striatal mechanisms and pathogenesis of parkinsonian signs and motor complications. Ann Neurol. 2000;47(Suppl 1):122-9.

7. Cohen-Cory S, Kidane AH, Shirkey NJ, Marshak S. Brain-derived neurotrophic factor and the development of structural neuronal connectivity. Dev Neurobiol. 2010;70(5):271-88.

8. Dekundy A, Pietraszek M, Schaefer D, Cenci MA, Danysz W. Effects of group I metabotropic glutamate receptors blockade in experimental models of Parkinson's disease. Brain Res Bull. 2006;69:318-26.

9. Fabbrini G, Brotchie JM, Grandas F, Nomoto M, Goetz CG. Levodopainduced dyskinesias. Mov Disord. 2007;22:1379-89.

10. Gan J, Qi C, Liu ZG. Roles of $\mathrm{Ca}^{2+} /$ calmodulin-dependent protein kinase II in subcellular expression of striatal n-methyl-d-aspartate receptors in I-3, 4-dihydroxyphenylalanine-induced dyskinetic rats. Drug Design Development Therapy. 2015;9:2119-28.

11. Gravius A, Dekundy A, Nagel J, More L, Pietraszek M, Danysz W. Investigation on tolerance development to subchronic blockade of mGluR5 in models of learning, anxiety, and levodopainduced dyskinesia in rats. J Neural Transm. 2008;115:1609-19.

12. Gregoire L, Morin N, Ouattara B, Gasparini F, Bilbe G, Johns D, Vranesic I, sahasranaman S, GomezMancilla B, Di Paolo T. The acute antiparkinsonian and antidyskinetic effect of AFQ056, a novel metabotropic glutamate receptor type 5 antagonist, in L-Dopa-treated parkinsonian monkeys. park. Relat Disord. 2011;17:270-6.

13. Guillin O, Diaz J, Carroll P, Griffon N, Schwartz JC. Sokoloff, P. BDNF controls dopamine D3 receptor expression and triggers behavioural sensitization. Nature. 2001;411(6833):86-9. 
14. Ham S, Lee YI, Jo M, Kim H, Kang H, Jo A, Lee GH, Mo YJ, Park SC, Lee YS, Shin JH, Lee YJ. Hydrocortisone-induced parkin prevents dopaminergic cell death via creb pathway in parkinson's disease model. Sci Rep. 2017;7:525.

15. Henry B, Crossman AR, Brotchie JM. Characterization of enhanced behavioral responses to L-DOPA following repeated administration in the 6-hydroxydopamine-lesioned rat model of Parkinson's disease. Exp Neurol. 1998;151:334-42.

16. Huang YX, Luo WF, Li Dan, Hu WD, Liu CF. CSC counteracts L-DOPA-induced overactivity of the corticostriatal synaptic ultrastructure and function in 6-OHDA-lesioned rats. Brain research. 2011;1376:113-21.

17. Huang YX, Shu HY, Li L, Zhen TL, Zhao JY, Zhou XJ, Luo WF. L-DOPA-Induced Motor Impairment and Overexpression of Corticostriatal Synaptic Components Are Improved by the mGluR5 Antagonist MPEP in 6-OHDA-Lesioned Rats. ASN neuro. 2018. doi:.

18. Johnston TH, Fox SH, Mclldowie MJ, Piggott MJ, Brotchie JM. Reduction f L-DOPA-induced dyskinesia by the selective metabotropic glutamate receptor 5 antagonist 3-[(2-methyl-1,3-thiazol-4yl)ethynyl]pyridine in the 1-methyl-4-phenyl-1,2,3,6-tetrahydropyridine-lesioned macaque model of Parkinson's disease. J Pharmacol Exp Therap. 2010;333:865-73.

19. Klockgether T, Turski L. Toward an understanding of the role of glutamate in experimental parkinsonism: agonist-sensitive sites in the basal ganglia. Ann Neurol. 1993;34:585-93.

20. Levandis G, Bazzini E, Armentero MT, Nappi G, Blandini F. Systemic administration of an mGluR5 antagonist, but not unilateral subthalamic lesion, counteracts I-DOPA-induced dyskinesias in a rodent model of Parkinson's disease. Neurobiol Dis. 2008;29:161-8.

21. Litim N, Morissette M, Di PT. Metabotropic glutamate receptors as therapeutic targets in parkinson's disease: an update from the last 5 years of research. Neuropharmacology. 2017;115:166-79.

22. Lundblad M, Andersson M, Winkler C, Kirik D, Wierup N, Cenci MA. Pharmacological validation of behavioural measures of akinesia and dyskinesia in a rat model of Parkinson's disease. EurJNeurosci. 2002;15:120-32.

23. Mela F, Marti M, Dekundy A, Danysz W, Morari M, Cenci MA. Antagonism of metabotropic glutamate receptor type 5 attenuates I-DOPA-induced dyskinesia and its molecular and neurochemical correlates in a rat model of Parkinson's disease. J Neurochem. 2007;101:483-97.

24. Meldrum BS. Glutamate as a neurotransmitter in the brain: review of physiology and pathology. J Nutr. 2000;130:1007S-1015S.

25. Morin N, Gregoire L, Gomez-Mancilla B, Gasparini F, Di Paolo T. Effect of the metabotropic glutamate receptor type 5 antagonists MPEP and MTEP in parkinsonian monkeys. Neuropharmacology. 2010;58:981-6.

26. Morin N, Gregoire L, Morissette M, Desrayaud S, Gomez-Mancilla B, Gasparini F, Di Paolo T. MPEP, an mGlu5 receptor antagonist, reduces the development of I-DOPA-induced motor complications in de novo parkinsonian monkeys: biochemical correlates. Neuropharmacology. 2013a;66:355-64. 
27. Morin N, Morissette M, Gregoire L, Gomez-Mancilla B, Gasparini F, Di Paolo T. Chronic treatment with MPEP, an mGlu5 receptor antagonist, normalizes basal ganglia glutamate neurotransmission in LDOPA-treated parkinsonian monkeys. Neuropharmacology. 2013b;73:216-31.

28. Morin N, Morissette M, Gregoire L, Gomez-Mancilla B, Gasparini F, Di Paolo T. Chronic treatment with MPEP, an mGlu5 receptor antagonist, normalizes basal ganglia glutamate neurotransmission in IDOPA-treated parkinsonian monkeys. Neuropharmacology. 2013c;73:216-31.

29. Oh JD, Chase TN. Glutamate-mediated striatal dysregulation and the pathogenesis of motor response complications in Parkinson's disease. Amino Acids. 2002;23:133-9.

30. Ouattara B, Gasparini F, Morissette M, Gregoire L, Samadi P, Gomez- Mancilla B, Di Paolo T. Effect of L-Dopa on metabotropic glutamate receptor 5 in the brain of parkinsonian monkeys. $J$ Neurochem. 2010;113:715-24.

31. Ouattara B, Gregoire L, Morissette M, Gasparini F, Vranesic I, Bilbe G, Johns DR, Rajput A, Hornykiewicz O, Rajput AH, Gomez-Mancilla B, Di Paolo T. Metabotropic glutamate receptor type 5 in levodopa-induced motor complications. Neurobiol Aging. 2011;32:1286-95.

32. Park H, Poo MM. Neurotrophin regulation of neural circuit development and function. Nat Rev Neurosci. 2013;14(1):7-23.

33. Paxinos G, Watson C. The Rat Brain in Stereotaxic Coordinates. 2nd ed. New York: Academic Press; 1986.

34. Platt SR. The role of glutamate in central nervous system health and disease - a review. Vet J. 2007;173:278-86.

35. Rascol O, Fox S, Gasparini F, Kenney C, Paolo TD, Gomez-Mancilla B. Use of metabotropic glutamate 5-receptor antagonists for treatment of levodopa-induced dyskinesias. Parkinsonism Relat Disord. 2014;20(9):947-56.

36. Rylander D, Iderberg H, Li Q, Dekundy A, Zhang J, Li H, Baishen R, Danysz W, Bezard E, Cenci MA. A mGluR5 antagonist under clinical development improves L-DOPA-induced dyskinesia in parkinsonian rats and monkeys. Neurobiol Dis. 2010;39:352-61.

37. Samadi P, Gregoire L, Morissette M, Calon F, Hadj Tahar A, Dridi M, Belanger N, Meltzer LT, Bedard PJ, Di Paolo T. mGluR5 metabotropic glutamate receptors and dyskinesias in MPTP monkeys. Neurobiol Aging. 2008;29:1040-51.

38. Yang X, Zhu Z, Ding X, Wang X, Cui G, Hua F, Xiang J. CaMKII inhibition ameliorated levodopainduced dyskinesia by downregulating tyrosine hydroxylase activity in an experimental model of parkinson's disease. Brain Res. 2018;1687:66-73.

39. Yuqin Z, Lifei Q, Wen X, Xiaoying W, Huang L, Wei X, Kedan C, Yu L. Paeoniflorin attenuates cerebral ischemia-induced injury by regulating $\mathrm{Ca}^{2+} / \mathrm{CaMKII} / \mathrm{CREB}$ signaling pathway. Molecules. 2017;22(3):359.

40. Zhang X, Andren PE, Svenningsson P. Repeated I-dopa treatment increases c-fos and BDNF mRNAs in the subthalamic nucleus in the 6-OHDA rat model of parkinson's disease. Brain Res. 2006;1095(1):207-10. 
41. Zhang SF, Xie CL, Wang Q, Liu ZG. Interactions of CaMKII with dopamine D2 receptors: roles in levodopa-induced dyskinesia in 6-hydroxydopamine lesioned parkinson's rats. Sci Rep. 2014;4:6811.

\section{Figures}

(a)

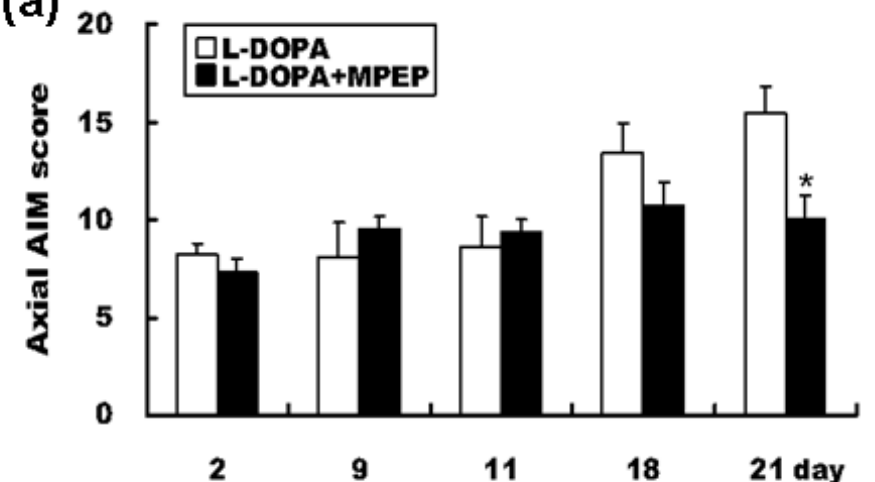

(c)

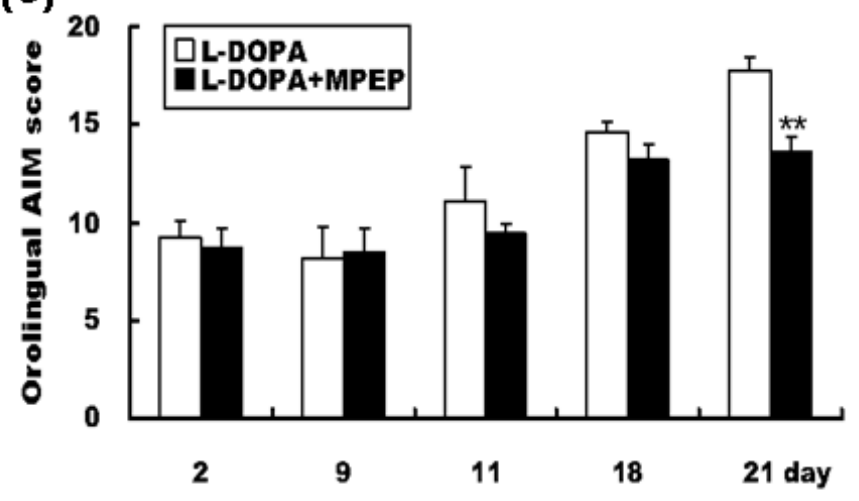

(b)

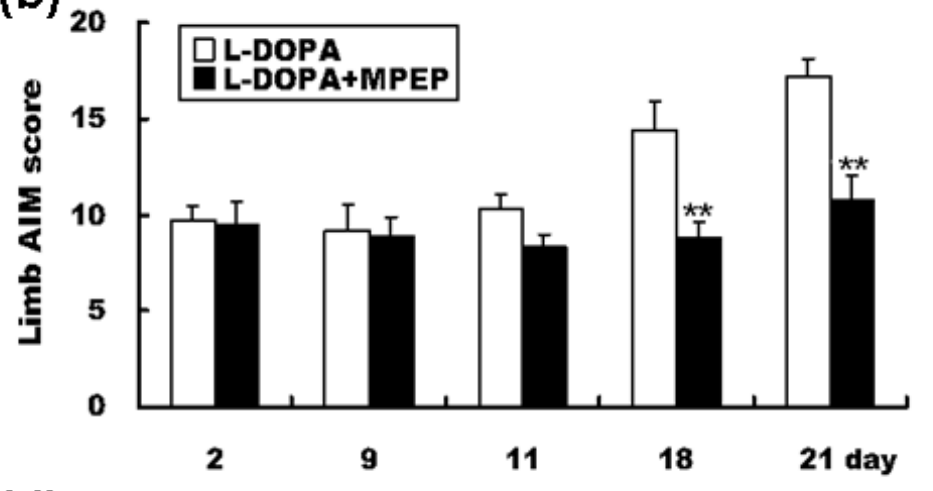

(d)

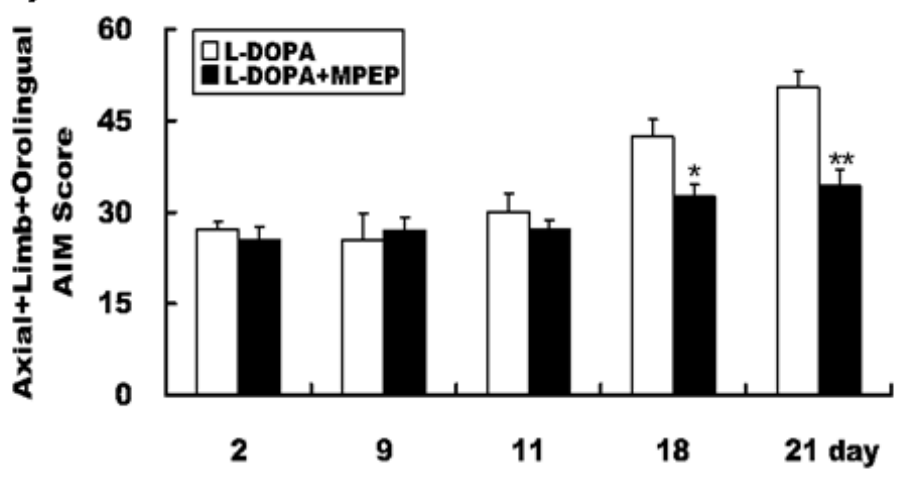

Figure 2

Effects of MPEP on L-DOPA-induced AIM in 6-OHDA-lesioned rats. AIM scores were recorded at 2, 9, 11, 18 and 21 days after L-DOPA application. Rats were daily treated with L-DOPA alone or with MPEP 30 min before L-DOPA for 3 weeks. Axial AIM scores (a), Limb AIM scores (b) , orolingual AIM scores (c) , and total (axial, limb, orolingual) AIM scores (d), were recorded shown at time points as mentioned above. Data were obtained from 10 animals for each group, and are shown as mean $\pm S E M$. ${ }^{*} P<0.05$, ${ }^{* * P}<0.01$ versus the L-DOPA treated group. 


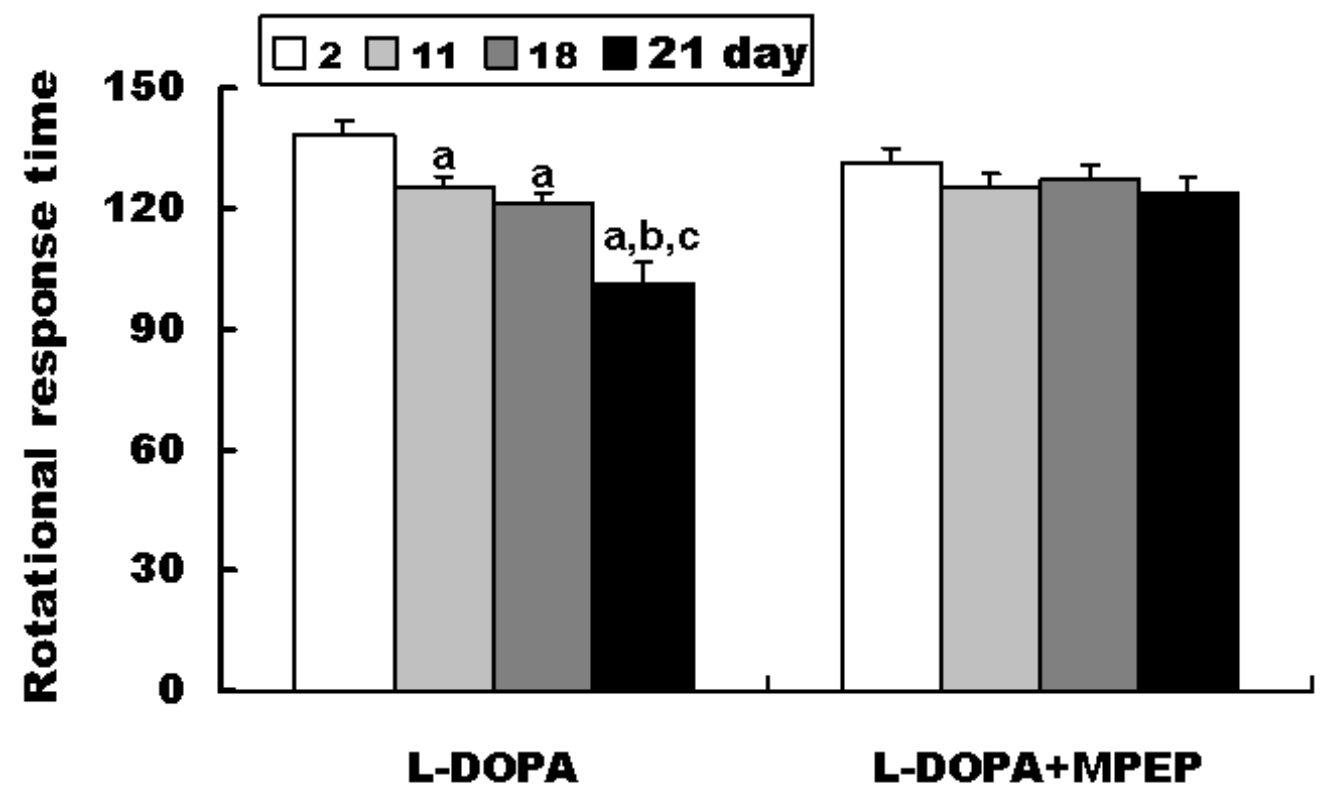

Figure 4

Effect of coadministration of L-DOPA with MPEP on the duration of the rotational response during the 21 days injection period. On each day, MPEP was given orally 30 min prior to L-DOPA challenge for 21 days. Data are reported as mean \pm SEM, from 10 animals. a $P<0.05$ vs.day 2; $b P<0.05$ vs. day 11 and $c P<0.05$ vs.day 18 , respectively. 
(a)
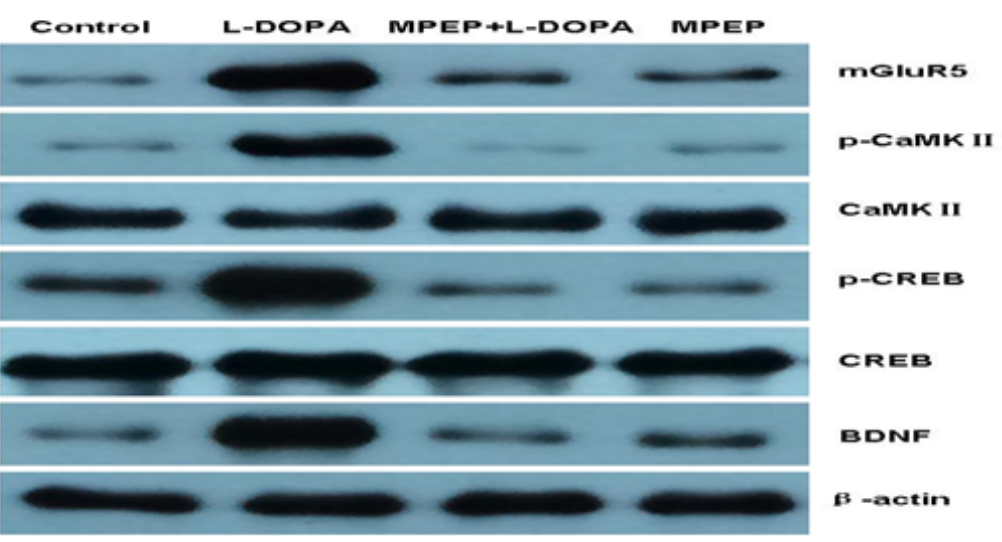

(b)

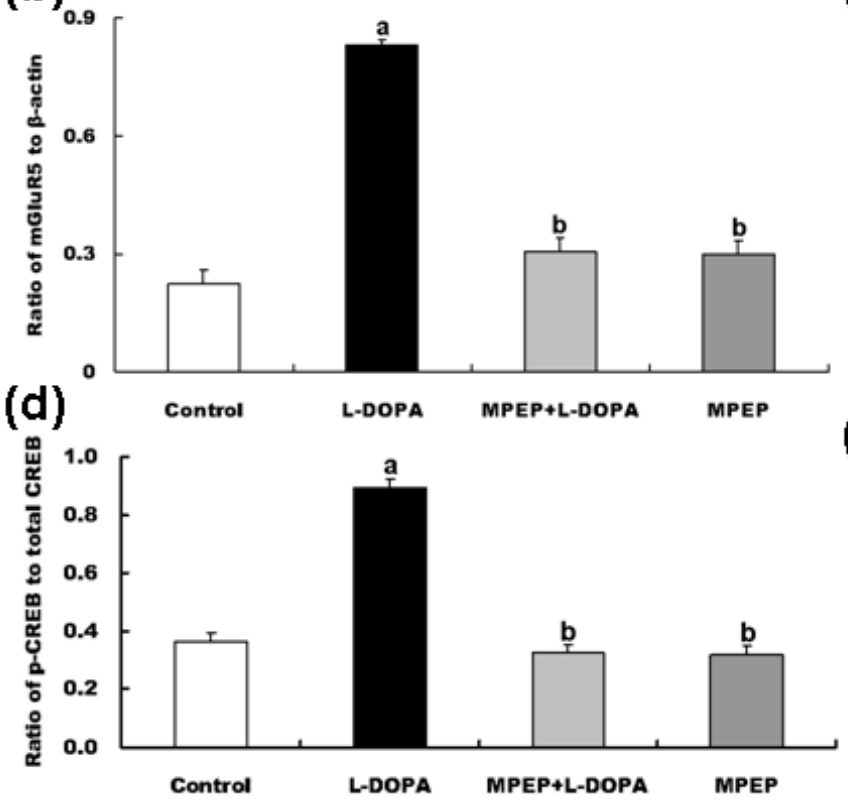

(c)

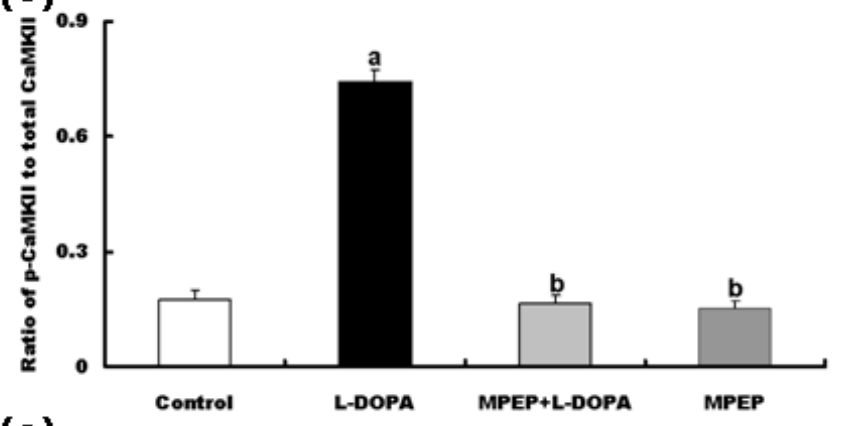

(e)

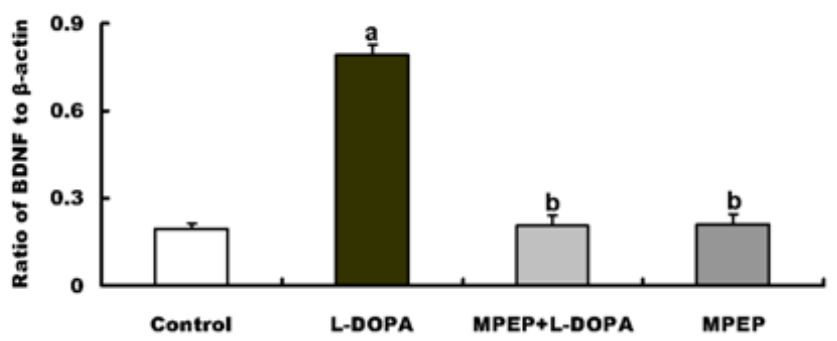

Figure 6

MPEP reversed the L-DOPA induced increase of striatal mGluR5, CaMKII, CREB and BDNF expression in lesioned side. The proteins were analyzed from lesioned striatum of the control group (saline group), the L-DOPA group, the L-DOPA plus MPEP group, or the MPEP group,. Data were obtained from 4 animals for each group, and are presented as mean \pm SEM. (a), representative blots; (b), (c), (d) and (e), quantitative analysis. a $\mathrm{P}<0.01$ versus the control group, $\mathrm{b} P<0.01$ versus the $\mathrm{L}-\mathrm{DOPA}$ treated group, respectively. 

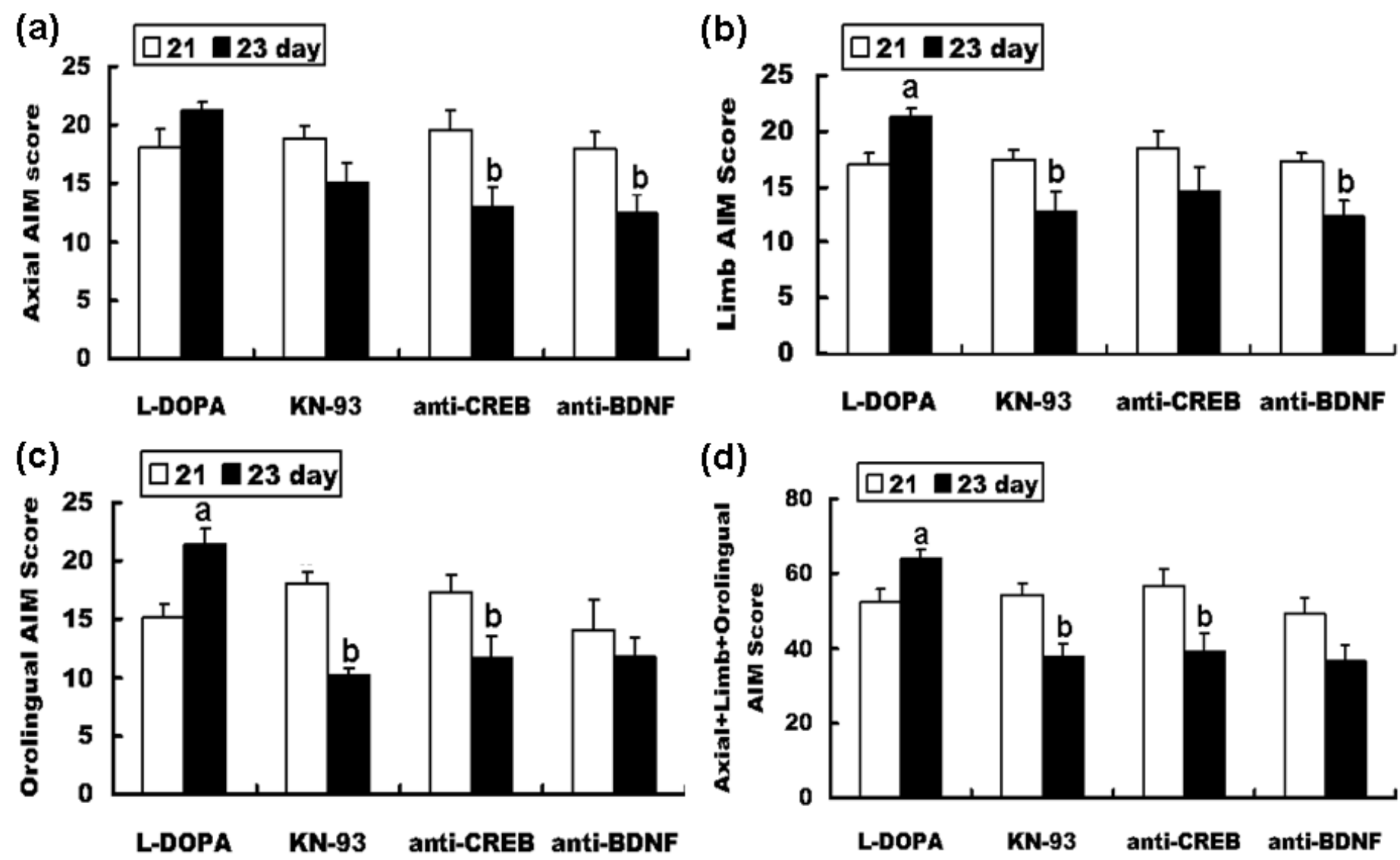

Figure 8

Effects of KN-93, anti-CREB, anti-BDNF attenuates L-DOPA-induced AIM in 6-OHDA-lesioned rats. AIM scores were recorded at 21 and 23 days after drug application. Rats were daily treated with L-DOPA to 21 days, the specific CaMKII inhibitor KN-93, anti-CREB or anti-BDNF intrastriatal administration at the 22 day, at 23 day, rats were treated with L-DOPA. Axial AIM scores (a), Limb AIM scores (b) , orolingual AIM scores (c) , and total (axial, limb, orolingual) AIM scores (d), were recorded shown at time points as mentioned above. Data were obtained from 10 animals for each group, and are shown as mean \pm SEM. a $P<0.05$, versus the 21 day with the L-DOPA-treated group, $b P<0.05$ versus the 21 day with the KN-93, antiCREB or anti-BDNF treated groups, respectively. 
(a)

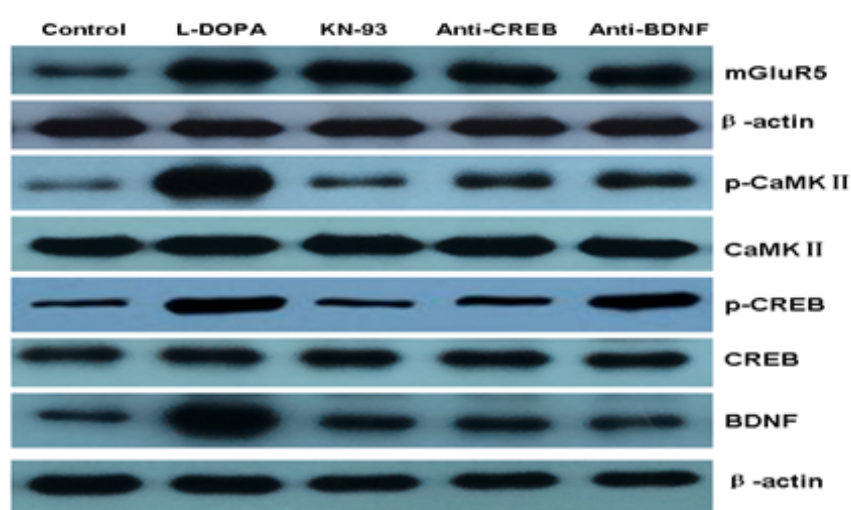

(b)

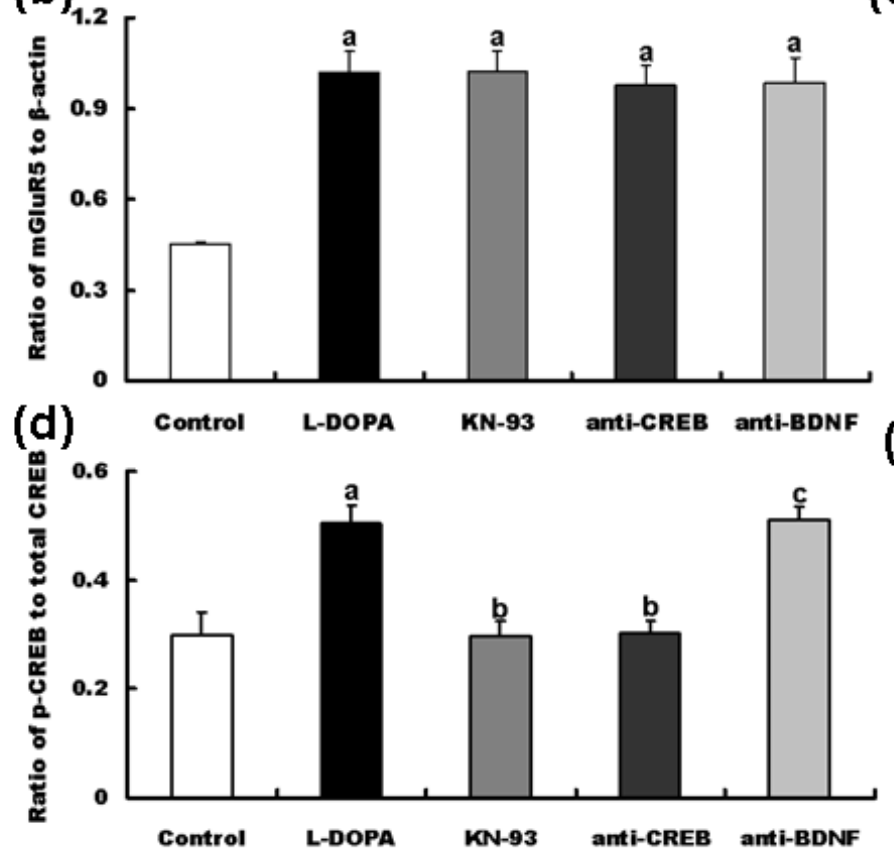

(c)
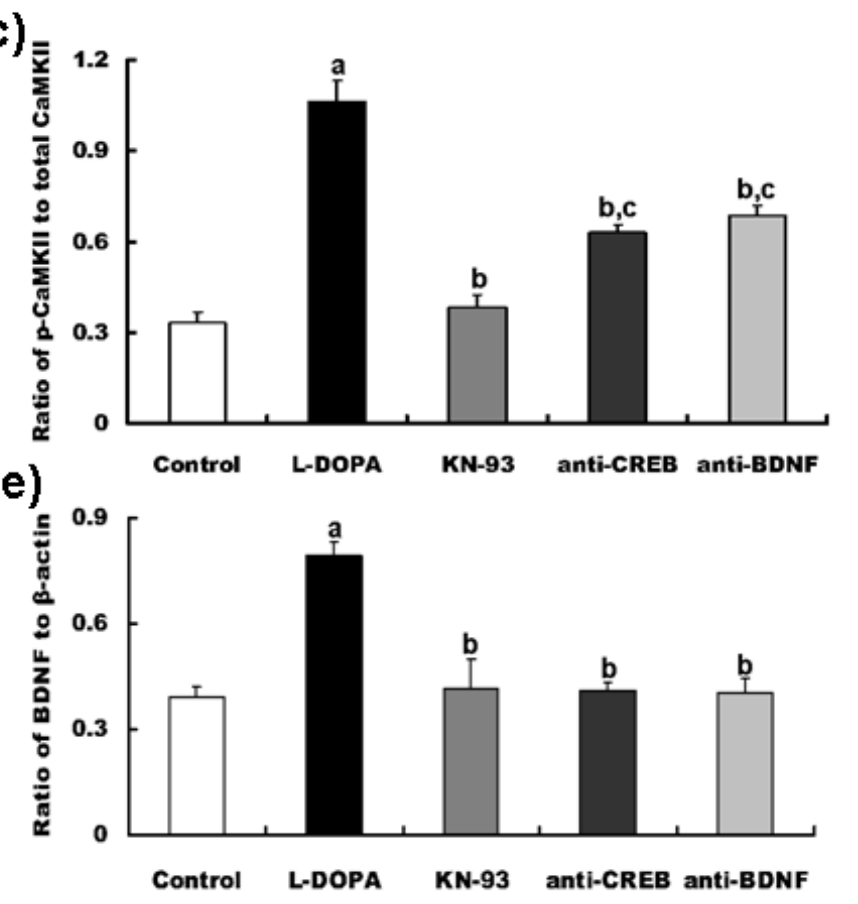

Figure 10

KN-93, anti-CREB, anti-BDNF affects L-DOPA-induced striatal CaMKII/CREB/BDNF expression in lesioned side. The proteins were analyzed from lesioned striatum of the control group (saline group), the L-DOPA group, KN-93 group, anti-CREB group, or the anti-BDNF group. Data were obtained from 4 animals for each group, and are presented as mean \pm SEM. (a), representative blots; (b), (c) and (d), quantitative analysis. a $P<0.01$ versus the control group, $b P<0.01$ versus the $L-D O P A$ treated group, $c P<0.01$ versus the $\mathrm{KN}-93$, or anti-CREB treated groups, respectively. 